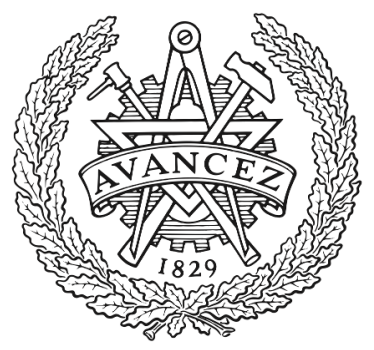

CHALMERS

UNIVERSITY OF TECHNOLOGY

\title{
Large-Eddy Simulation of the Flow Around Simplified Car Model
}

Downloaded from: https://research.chalmers.se, 2023-04-26 13:15 UTC

Citation for the original published paper (version of record):

Krajnovic, S., Davidson, L. (2004). Large-Eddy Simulation of the Flow Around Simplified Car

Model. SAE Technical Papers. http://dx.doi.org/10.4271/2004-01-0227

N.B. When citing this work, cite the original published paper. 


\section{Large-Eddy Simulation of the Flow Around Simplified Car Model}

Siniša Krajnović and Lars Davidson Department of Thermo and Fluid Dynamics, Chalmers University Of Technology, SE-412 96 Göteborg, Sweden

Copyright (C) 2004 Society of Automotive Engineers, Inc.

\begin{abstract}
A hypothesis of using lower Reynolds number large eddy simulation (LES) to simulate the flow around ground vehicle at higher Reynolds number was tested. The simulation was made of the flow around a simplified car model and the results were compared with the experimental data. It has been found that the level of Reynolds number, if sufficiently high, has small influence on the results in highly separated flows around cars. This raises hope that the LES of the external car flow is feasible.
\end{abstract}

\section{INTRODUCTION}

More than thirty years of computational fluid dynamics (CFD) made a huge contribution to our understanding of flows around ground vehicles. Although these turbulent flows are adequately described by the unsteady Navier-Stokes equations, they are rarely solved due to high computational cost. Instead the simplified Reynolds Averaged Navier-Stokes (RANS) equations are solved. These equations are time-averaged and thus they provide us only with the mean information of the flow and the unsteady information is lost. The Reynolds stresses in these equations are modeled with a turbulence model. Thus our success to give a true representation of the mean flow around a vehicle is dependent on the turbulence model used. Unfortunately it is difficult to define a model that can accurately represent the Reynolds stresses in the regions of the separated flow such as a wake behind e.g. a car (Later in the paper we shall demonstrate in the example of the flow studied here, the main difficulties in constructing 'the universal' turbulence model for automobile ap- plications.). The consequence is that the mean surface pressure cannot be accurately predicted leading to poor representation of the mean aerodynamic forces.

Increase in the computer power in recent years have made time-dependent simulations (of moderate Reynolds numbers flows) possible where large flow structures are directly computed and only the influence of the structures smaller than the computational cells are modeled. This is done in a technique called large eddy simulation (LES). LES of the external vehicle flow is considered computationally infeasible. Before we reject making LES of such flows let us discuss the main obstacle of such a simulation i.e. the high Reynolds number. The resolution requirement of a wall bounded LES are based on experimental observations and DNS studies of boundary layers. These studies exposed the so-called buffer region as the part of the boundary layer where most of the turbulence energy is produced and dissipated. "This region is characterized by a bursting process, during which low-speed fluid (provided in the form of streaks) is flung outward from the wall, generating most of the turbulence production in the boundary layer." [1]. Thus accurate representation of these vortices is of great importance for accurate prediction of the flow.

The problem with these structures is that they decrease in size as we increase the Reynolds number. Although there is no exact resolution requirement for their representation, most LES practitioners consider a resolution sufficient when it is expressed in wall units (e.g. $y^{+}=u_{\tau} y / \nu$ in the wall-normal direction) as $y^{+}<1$, $\Delta x^{+} \simeq 50-150$ and $\Delta z^{+} \simeq 15-40$ in the wall-normal, the streamwise and the spanwise directions, respec- 
tively. Krajnović and Davidson [2] demonstrated that such a resolution would lead to computational mesh containing more than $6 \times 10^{8}$ cells in the near-wall region only for very simple vehicle geometry at the Reynolds number of $5 \times 10^{6}$ typical for a passenger car.

Let us go back to our question of resolution requirement in a LES of a passenger car. Do we really need to have the same resolution in the near wall region of a car as in the one of a flat plate or a plane channel? The answer to this question is given in the difference between these two flows. The former is characterized by the separated regions that dominate the flow whereas the turbulence production in the latter comes from the near wall coherent structures. Thus the answer is that the resolution requirements in the car-flow LES can probably be relaxed in some extent compared to the one in flat-plate LES. The question of real resolution requirements in a car-flow LES can only be answered with extensive mesh refinement studies.

The second issue needed to be investigated is how large the Reynolds number that needs to be in the simulations for accurate representation of the real flow. This can again be related to the character of the flow. The Reynolds number defines the boundary layer thickness which in turn defines the separation of the flow. This is of course true for a smooth surface but what happens if the separation of the flow is already defined by the geometry (as it is the case in many parts of the flow around a car such as at mirrors, windshield wipers, body underside, in the wheel wells or at the rear end of many cars)? The goal of this paper is to investigate the influence of reducing the Reynolds number in LES. If the results of the simulations at lower Reynolds number are in agreement with experimental data at higher Reynolds number, it is likely that the flow around vehicles becomes Reynolds number independent for high Reynolds numbers. In this case the flow around vehicles at high Reynolds numbers can be represented with LES using a lower Reynolds number.

\section{SIMPLIFIED CAR MODEL}

The generic car body defined by Ahmed [3] has been used in several experimental $[3,4,5,6,7,8]$ and numerical studies $[9,10]$. A slightly modified version of this body was also studied in [11, 12, 13, 14, 2, 15]. In these studies the angle of the slanted back was zero making this body a generic bus rather than a car. The shape of the original body [3] is shown in Fig. 1. The rear end of this model is simplification of a so-called fastback rear end such as on a Volkswagen Golf I. Although several slant angles were used in the experimental studies, two angles, $\alpha=25^{\circ}$ and $\alpha=35^{\circ}$, are overrepresented. These angles are often chosen for studies because they are surrounding the critical angle of $\alpha=30^{\circ}$ where the flow changes character (see e.g. [3]). At the angle of $\alpha=30^{\circ}$ the flow remains partially attached whereas an increase of this angle produces fully separated flow over the rear slant.

Most of the numerical simulations of these flows are steady RANS simulations [9, 10]. It was observed that RANS simulations performed relatively well for the $\alpha=35^{\circ}$ angle case and poor for the $\alpha=25^{\circ}$ angle case. A possible reason for failure of turbulence models to predict the $\alpha=25^{\circ}$ angle case could be much higher level of unsteadiness in this flow. According to Durant et al. [10] "Most RANS turbulent models would underestimate or miss the separation entirely and return too optimistic flow conditions. Other models, which have been optimized to accurately predict the separation onset, fail in the massively separated regions." One LES was also presented by Hinterberger et al. [10]. Although they used $18.5 \times 10^{6}$ computational cells, their results were in a poor agreement with the experiments of Lienhart and Becker [7].

\section{GEOMETRY AND NUMERICAL DETAILS}

The geometry of the body is given in Fig. 1. All the geometric quantities are normalized with the body height, $H$, equal to $0.288 \mathrm{~m}$. The values of the geometric quantities are $L / H=3.625, l_{r} / H=2.119, B / H=1.351$. The front part is rounded with a radius of $R / H=0.347$. The flow with the rear body slant angle $\alpha=25^{\circ}$ is considered in this paper since it was found difficult to predict in previous RANS and LES simulations [10]. This body is placed in the channel with cross section of $6.493 \mathrm{H} \times 4.861 \mathrm{H}$ (width $\times$ height). The cross section of this channel is identical with the open test section of the wind tunnel used in the experiments of Lienhart and Becker [7]. The front face of the body is located at the distance of $7.3 \mathrm{H}$ from the channel inlet and the downstream length between the rear face of the body and the channel outlet is $21 \mathrm{H}$. The body is lifted from the floor producing the ground clearance of $0.174 \mathrm{H}$, same as in the experiments. The Reynolds number, based on the incoming velocity $U_{\infty}$ and the car height $H$, of $R e=7.68 \times 10^{5}$ used in the experiments [7] was reduced to $R e=2 \times 10^{5}$. Krajnović and Davidson [2, 15] have already demonstrated LES of this lower Reynolds number case when the rear body slant angle $\alpha=0^{\circ}$ (generic bus body). We expect that the slanted rear end will produce a wider spectrum of turbulent scales that must be resolved in LES.

The wind tunnel in the experiments of Lienhart and Becker [7] produced the average turbulent intensity of $0.25 \%$. A uniform velocity profile constant in time was thus used as the inlet boundary condition in our LES. The convective boundary condition $\partial \bar{u}_{i} / \partial t+$ $U_{c}\left(\partial \bar{u}_{i} / \partial x\right)=0$ was used at the downstream boundary. 


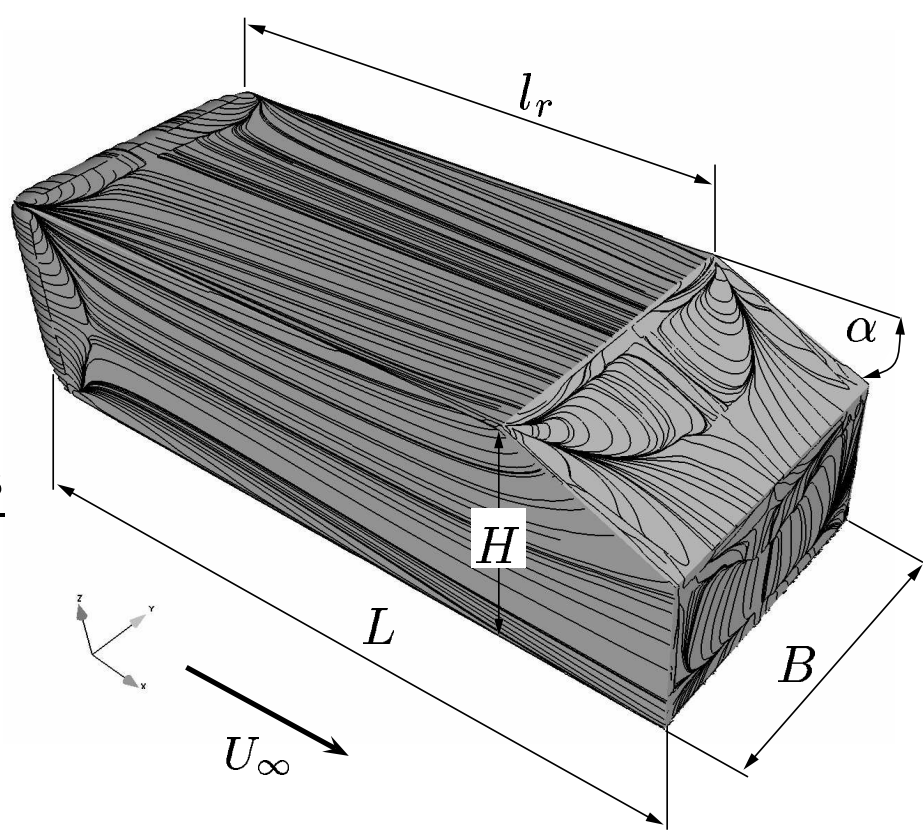

Figure 1: Geometry of the vehicle body. Time-averaged trace lines are shown on the body surface. View from behind and lateral side.

Here, $U_{c}$ was set equal to the incoming mean velocity, $U_{\infty}$. The lateral surfaces and the ceiling were treated as slip surfaces using symmetry conditions $(\partial \bar{u} / \partial z=$ $\partial \bar{w} / \partial z=\bar{v}=0$ for the lateral sides and $\partial \bar{u} / \partial z=$ $\partial \bar{v} / \partial z=\bar{w}=0$ for the ceiling). This boundary condition is different from the experimental one where the test section had a floor but no sides or ceiling. The consequence of this boundary condition is that the flow across the lateral sides and the ceiling is permitted in the experiment but not in the simulation resulting in different 'effective' blocking of the cross section. No-slip boundary conditions were used on the surface of the body and on the channel floor.

Computations on two different computational grids containing 3.5 and 9.6 millions nodes, respectively, were made. An additional computation on a grid containing 16.7 million nodes is ongoing during the writing of this paper. The time step was $1 \times 10^{-4}$, giving a maximum CFL number of approximately 0.9 . The averaging time, $t U_{\infty} / H$, in the simulations was $38.2(110,000$ time steps).

\section{GOVERNING EQUATIONS AND SUBGRID-SCALE MODELING}

The governing LES equations are the incompressible Navier-Stokes and the continuity equations filtered with the implicit spatial filter of characteristic width $\Delta$ ( $\Delta$ is the grid resolution in this work):

$$
\frac{\partial \bar{u}_{i}}{\partial t}+\frac{\partial}{\partial x_{j}}\left(\bar{u}_{i} \bar{u}_{j}\right)=-\frac{1}{\rho} \frac{\partial \bar{p}}{\partial x_{i}}+\nu \frac{\partial^{2} \bar{u}_{i}}{\partial x_{j} \partial x_{j}}-\frac{\partial \tau_{i j}}{\partial x_{j}}
$$

and

$$
\frac{\partial \bar{u}_{i}}{\partial x_{i}}=0
$$

Here, $\bar{u}_{i}$ and $\bar{p}_{i}$ are the resolved velocity and pressure, respectively, and the bar over the variable denotes filtering.

These equations are derived applying a filtering operation

$$
\bar{f}\left(x_{i}\right)=\int_{\Omega} f\left(x_{i}^{\prime}\right) G\left(x_{i}, x_{i}^{\prime}\right) d x_{i}^{\prime}
$$

on the Navier-Stokes and the continuity equations [16]. Here $G$ is a top hat filter function and $\Omega$ represents the entire flow domain. The filtered variables in the governing Eqs. (1) and (2) are obtained implicitly through the spatial discretization.

The goal of the filtering is to decompose the fluid motion into a large-scale component that are resolved and the small subgrid scale (SGS). The influence of the small scales of the turbulence on the large energy carrying scales in Eq. (1) appears in the SGS stress tensor, $\tau_{i j}=\overline{u_{i} u_{j}}-\bar{u}_{i} \bar{u}_{j}$, which must be modeled. The algebraic eddy viscosity model originally proposed by Smagorinsky [17] is used in this paper for its simplicity and low computational cost. The Smagorinsky model represents the anisotropic part of the SGS stress tensor, $\tau_{i j}$, as:

$$
\tau_{i j}-\frac{1}{3} \delta_{i j} \tau_{k k}=-2 \nu_{s g s} \bar{S}_{i j}
$$

where $\nu_{s g s}=\left(C_{s} f \Delta\right)^{2}|\bar{S}|$ is the SGS viscosity,

$$
\bar{S}_{i j}=\frac{1}{2}\left(\frac{\partial \bar{u}_{i}}{\partial x_{j}}+\frac{\partial \bar{u}_{j}}{\partial x_{i}}\right)
$$

is the resolved rate-of-strain tensor and $|\bar{S}|=$ $\left(2 \bar{S}_{i j} \bar{S}_{i j}\right)^{\frac{1}{2}}$. $f$ in the expression for the SGS viscosity is the van Driest damping function

$$
f=1-\exp \left(\frac{y^{+}}{25}\right)
$$

Using this damping function, wall effects are partially taken into account by 'damping' the length scale $l=$ $C_{s} f \Delta$ near to the walls. The Smagorinsky constant, $C_{s}$, must be adjusted for different flows. The value of $C_{s}=0.1$ previously used for bluff-body flows $[18,19,20]$ and flow around simplified bus [2, 15] is used in this work. The filter width, $\Delta$, is defined in this work as $\Delta=\left(\Delta_{1} \Delta_{2} \Delta_{3}\right)^{1 / 3}$, where $\Delta_{i}$ are the computational cell sizes in three coordinate directions.

\section{NUMERICAL METHOD}

LES Eqs. (1) and (2) are discretized using a 3D finite volume method for solving the incompressible 
Navier-Stokes equations using a collocated grid arrangement [21]. Both convective and viscous plus subgrid fluxes are approximated by central differences of second-order accuracy. The time integration is done using the Crank-Nicolson second-order scheme. The SIMPLEC algorithm is used for the pressure-velocity coupling. The code is parallelized using block decomposition and the PVM and MPI message passing systems [22]. Additional details on this code can be found in [21] and [22].

\section{BEST PRACTICE GUIDELINES FOR LES OF THE VEHICLE FLOWS}

With knowledge that other researchers already failed to predict the considered flow with LES, we tried to define best practice guidelines that we followed in our simulations. These can be divided in four groups: computational mesh, fully developed flow, averaging time and choice of Reynolds number. In the following we shall describe our philosophy of making good LES of external vehicle flows.

FOCUS ON THE COMPUTATIONAL MESH Most of the LES are using structured grids. The authors experience is that the number of computational cells in this wall-bounded flows becomes easily too large in structured grids. Concentrating the available computational cells where they are mostly needed such as boundary layers and regions of separated flows is thus essential. This can be achieved using the blocking topology combined of the blocking structures such as $O$ and $C$ grids rather than only using $\mathrm{H}$ grids. Let us demonstrate one of these strategies used in this paper. The computational mesh in the symmetry plane is shown in Fig. 2.

The topology of the grid consists of an $O$ grid, with a thickness of $0.1 H$ and a $C$ grid around the $O$ grid (see Fig. 2). An additional 'dummy' car surface was made around the real car and the $O$ grid was projected on these two surfaces. The rest of the blocking structures was made using $H$ grids. This strategy resulted in the following distribution of the computational cells. The $O$ grid (i.e. the region in a belt of thickness $0.1 \mathrm{H}$ around the body) contained 4.4 and 7.5 million cells of totally 9.6 and 16.5 million cells in the medium and the fine grids, respectively. The region containing the $O$ and the $C$ grids together (i.e. the region in a belt of thickness $0.28 \mathrm{H}$ around a car) hold 6.3 and 10.8 million cells in the medium and the fine grids, respectively.

FULLY DEVELOPED FLOW The initial guess in a LES if often fluid at rest (as in this paper) or solution from RANS simulation. Before we start to rely on our instantaneous solutions and begin time averaging, we must ensure that our initial guess evolved to fully developed flow that we intended to study. This task is not trivial in a very complex flow around vehicles. Probably, the only way to be sure that the flow has developed is the 'a posteriori' one (i.e. after the entire simulation). Such a test could prove that the mean, RMS values and the spectral picture of the solution are not dependent on the position in time where we started sampling of the solution. As we need to start the time averaging before we can perform the 'a posteriori' test, some approximate method must be used to ensure that the characteristics of the flow are not changing. The authors monitored the time sequences equal the time required for the fluid
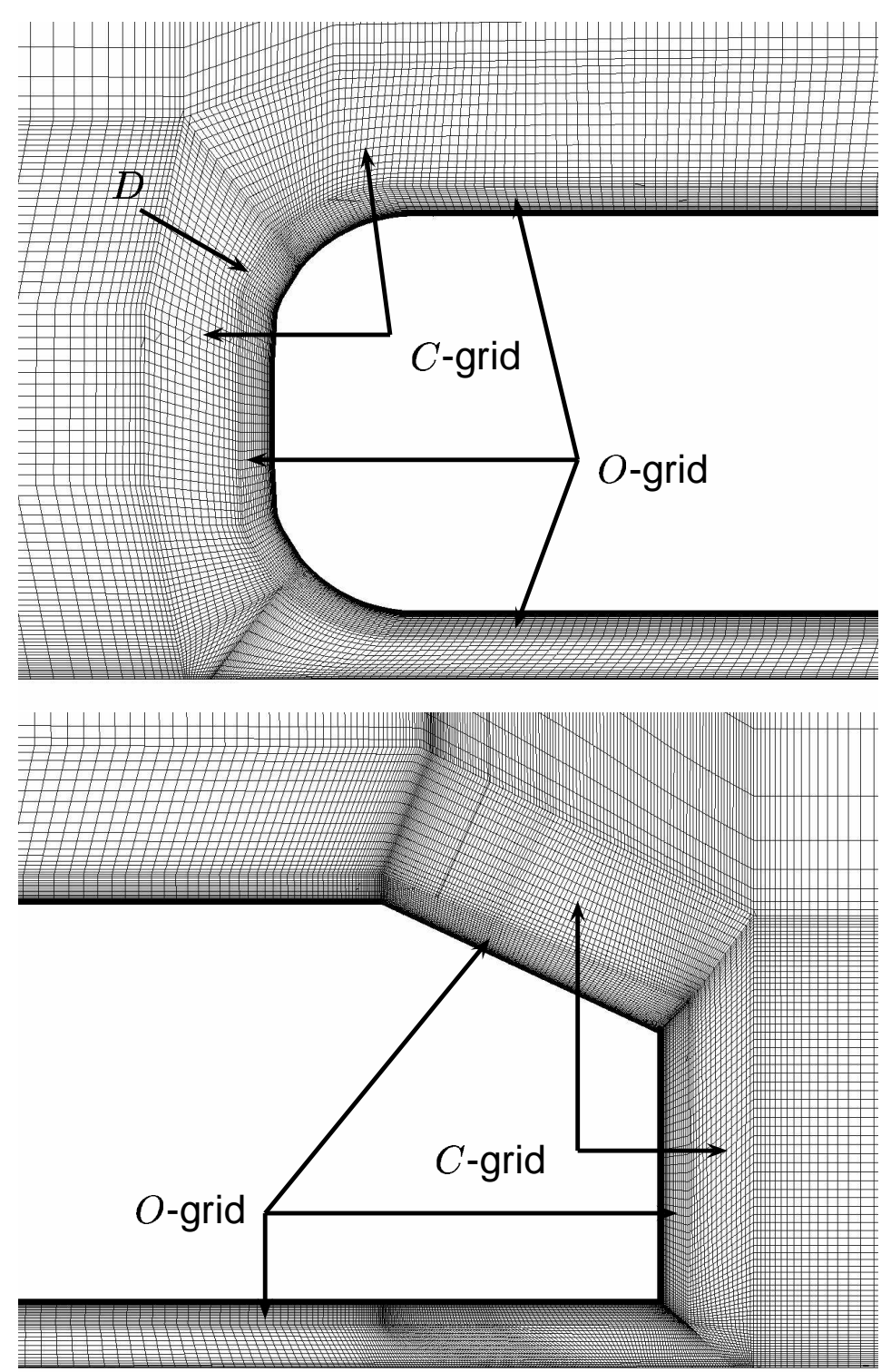

Figure 2: The computational mesh in symmetry plane. Above: the front part of the body; below: the rear part of the body. $D$ indicates the position of the dummy surface. View from the lateral side. 
particle to travel from the front to the rear face of the car body. We then computed the mean and the RMS values of the global quantities (aerodynamic forces) and local variables (velocity components and pressure in several points in and around the wake behind the body) for this time interval. The Fourier transforms of both global and local quantities were computed but because of the relatively short time sequences we could rely only on the high frequency events. The time averaging was started when there were no significant differences in the mean and the instantaneous information between two sequential time sequences.

AVERAGING TIME The time used for the averaging of the solution must be sufficiently long to produce the mean solution that is not a function of time. This can be a problem in LES where the size of the time step in the simulations is limited by the stability requirements and the resolution in time. It can be tempting to shorten the averaging time in the simulations when very small computational cells (i.e. small time steps) are used. This must be avoided and some kind of test should be used to find out if the averaging time was sufficient in the simulations. In the LES in this paper the symmetry of the flow around a symmetry plane ( $y=0$ plane) was used as a proof of long enough averaging time.

SIMULATING THE RIGHT REYNOLDS NUMBER Although the core of this paper is the use of a reduced Reynolds number $(R e)$ in LES compared to experimental we chose to place this assumption here. It remains to confirm our hypothesis on the following pages. As was already mentioned the flow around cars is dominated by the large coherent structures as a result of separations and the importance of resolving the fluid streaks in the near wall region is smaller than in attached flows. On the other hand reducing the Reynolds number makes it possible to resolve these coherent structures (although of a different size) whereas an attempt to resolve these structures (using the same computational grid) in a high $R e$ flow would result in failure due to poor resolution.

\section{RESULTS}

All the results presented in this paper are from the LES using the medium grid if not otherwise stated. Our interest was mainly based on the flow around the rear part of the body for which the experimental data by Lienhart and Becker [7] are available. Besides that, some qualitative results on the coherent structures are presented along the upstream part of the body. We have reduced the Reynolds number compared with the experimental one and there are no experimental data on the flow around the front of the body in [7]. Thus we don't know if the flow separates on the leading edge or remains at- tached at $R e=7.68 \times 10^{5}$. Therefore we have compared our results with another experiment where the Reynolds number was closer to the one in our LES.

INSTANTANEOUS FLOW The flow separates on the sharp edge between the roof of the body and the slant surface and vortices parallel with the line of separation are formed (see Fig. 3). As they are convected downstream, they are lifted forming hairpin vortices ( $\lambda$ in Fig. 4). At the same time the flow separates at two tilted edges between the slant and the side surfaces of the car model (Fig. 3). This results in two large counterrotating cone-like trailing vortices shown in Fig. 3 . We have also observed two pairs of thinner trailing vortices located close to the slant edge under the mantle of the large vortices (see Fig. 3). The large trailing vortices interact with smaller $\lambda$ structures mentioned above. They push the surrounding $\lambda$ vortices inward toward the center of the slant surface forcing them to change their axes from that parallel with the edge of separation (see Fig. 4).

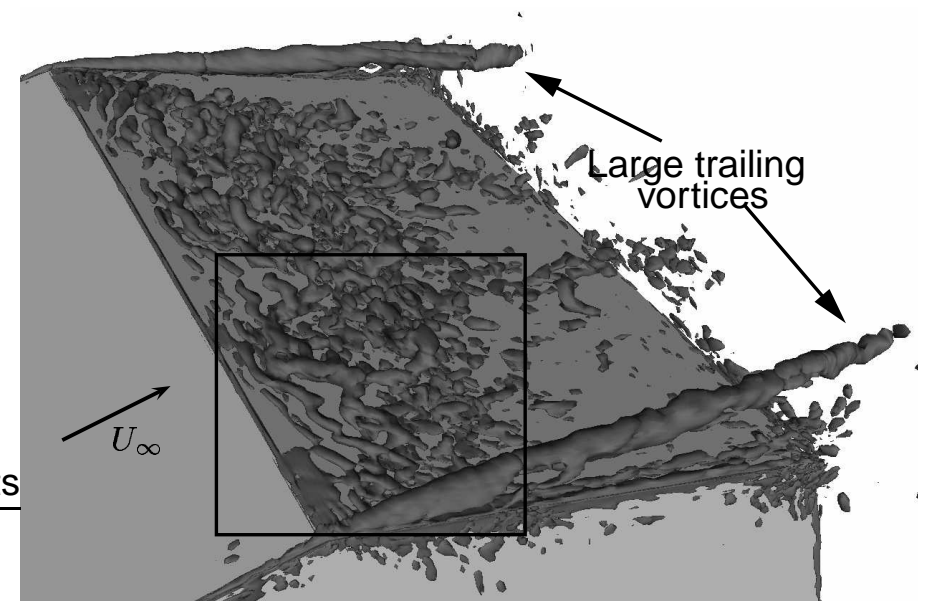

Figure 3: The isosurface of the instantaneous second invariant of the velocity gradient, $Q=3000$. The direction of the flow is from left to right. Note that only the rear slant surface is shown.

TIME-AVERAGED FLOW The character of the flow (i.e. attached or detached) around the front part of the body depends on the Reynolds number and the curvature of the front of the body. Normally the lower the Reynolds number the rounder leading edge is needed to prevent the separation. Unfortunately there are no experimental observations of this flow region at the high Reynolds number $\left(R e=7.68 \times 10^{5}\right)$. Thus we cannot judge the universalness of our results at our reduced Reynolds number. We observed three separated regions, one on the top and one on each lateral side of the car model. these are qualitatively in agreement with the observations by Sims-Williams and Dominy [4] at $R e=1.71 \times 10^{5}$ (see Fig. 5). Similar flow behavior at 
different curvature of the leading edges was observed in LES by Krajnović and Davidson [2, 15].

a)

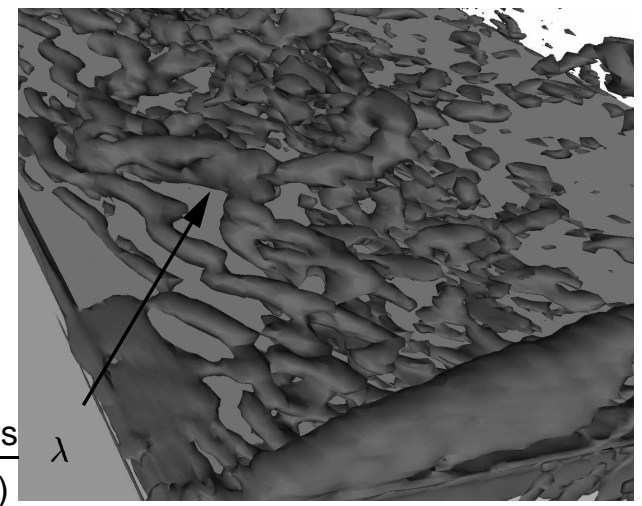

b)

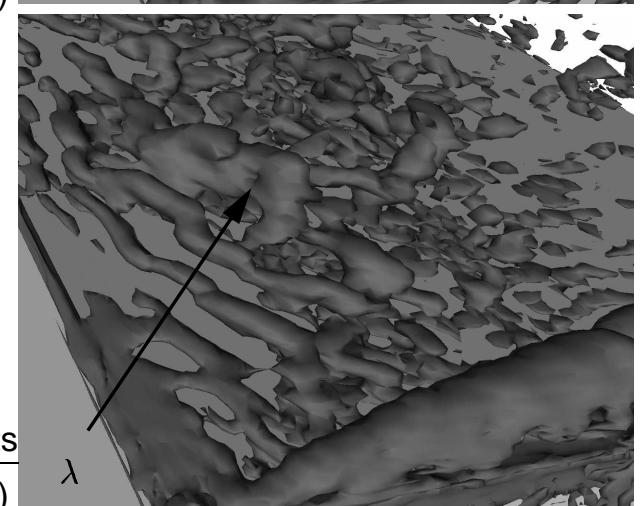

c)

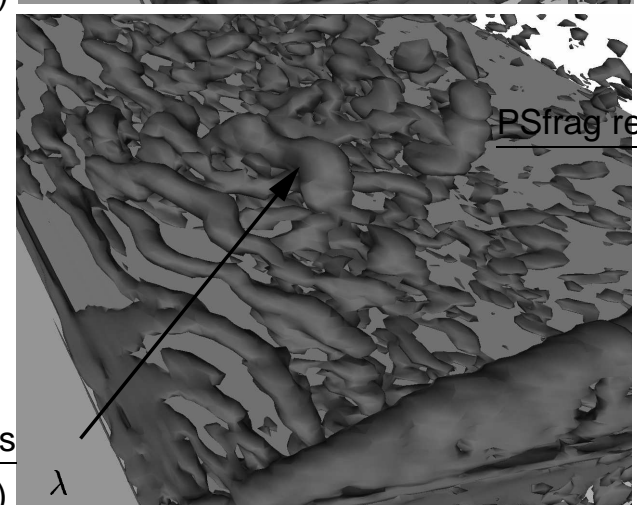

d)

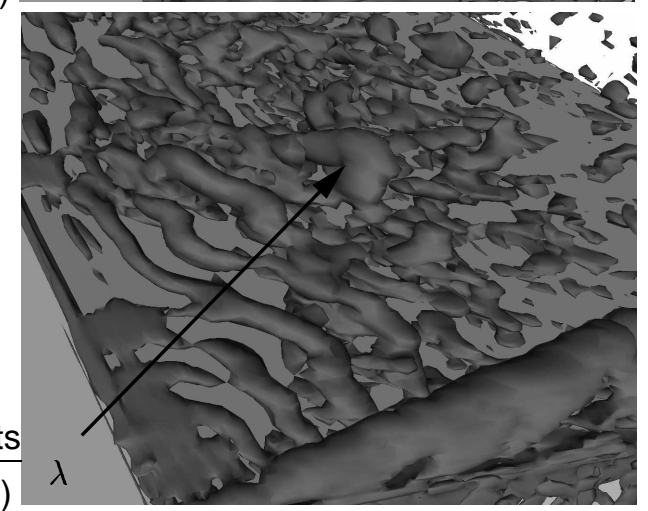

Figure 4: Zoom of Fig. 3. Figure a) shows the same time step as in Fig. 3. Figures b) - d) are the sequential time steps after the one in Fig. a). The dime difference between two pictures is $t U_{\infty} / H=0.05$.
As shown by Krajnović and Davidson [2, 15] for a similar body, the flow under the car model has a threedimensional character. The fluid from underneath the body traveling to the lateral sides forms two trailing vortices close to the lower side edges of the body (Fig. 6). The left (shown in Fig. 6) and the right vortices rotate clockwise and counterclockwise, respectively, similar to those in $[2,15]$. They stretch from approximately $x=-2.79 H$ to $x=0.35 H$ behind the body. As they move downstream, they first grow and later shrink in diameter (see Fig. 6).

When time-averaged the hairpin vortices on the rear slant surface (from Fig. 4) form a separation region

a)

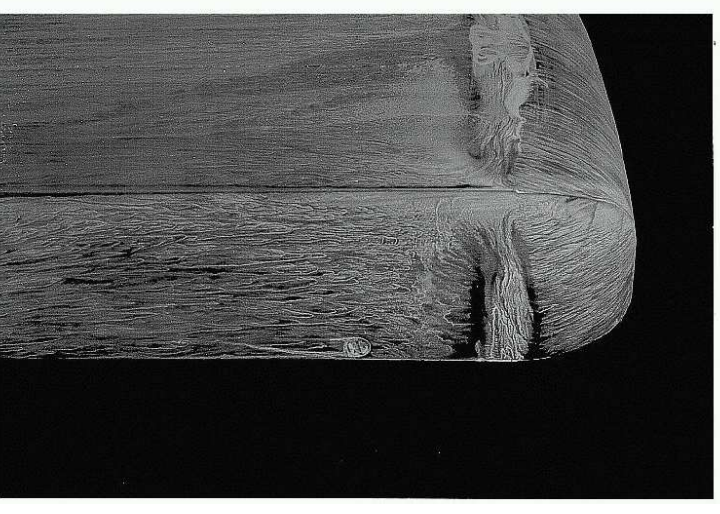

b)

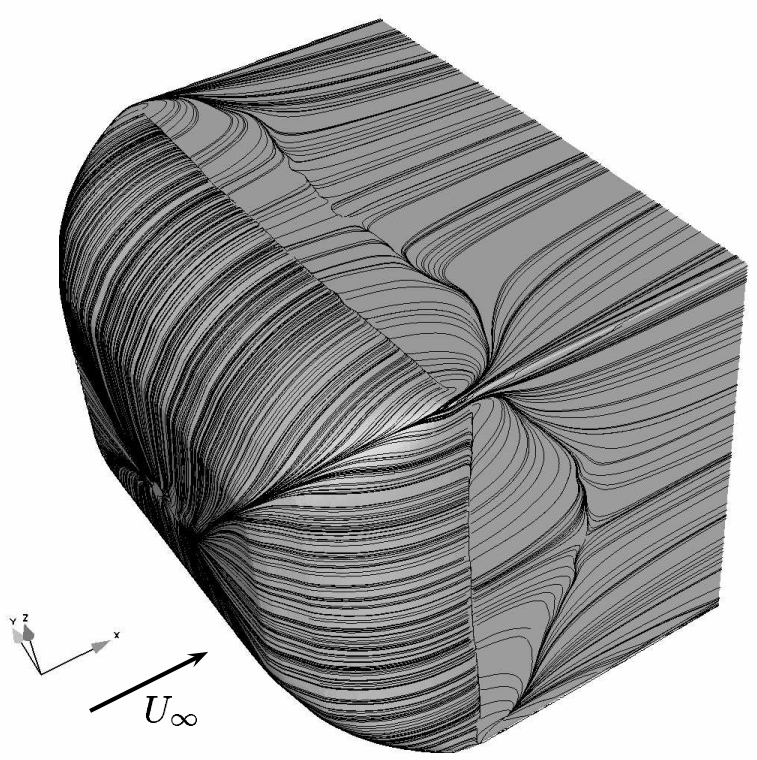

Figure 5: Comparison of a) oil-film visualization by Sims-Williams and Dominy [4] at $R e=1.71 \times 10^{5}$ with b) time-averaged trace lines on the surface of the body from LES. Flow is from right to left in Fig. a) and from left to right in Fig $b$ ). 


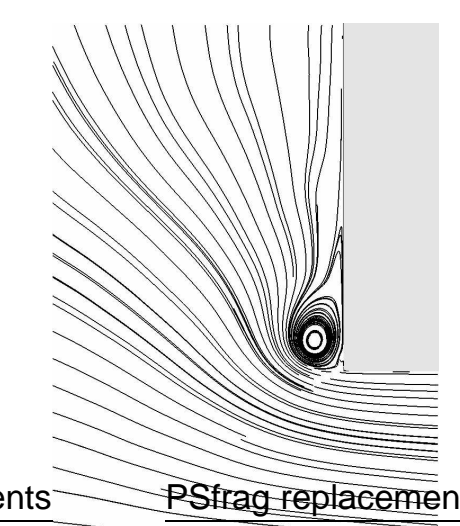

a)
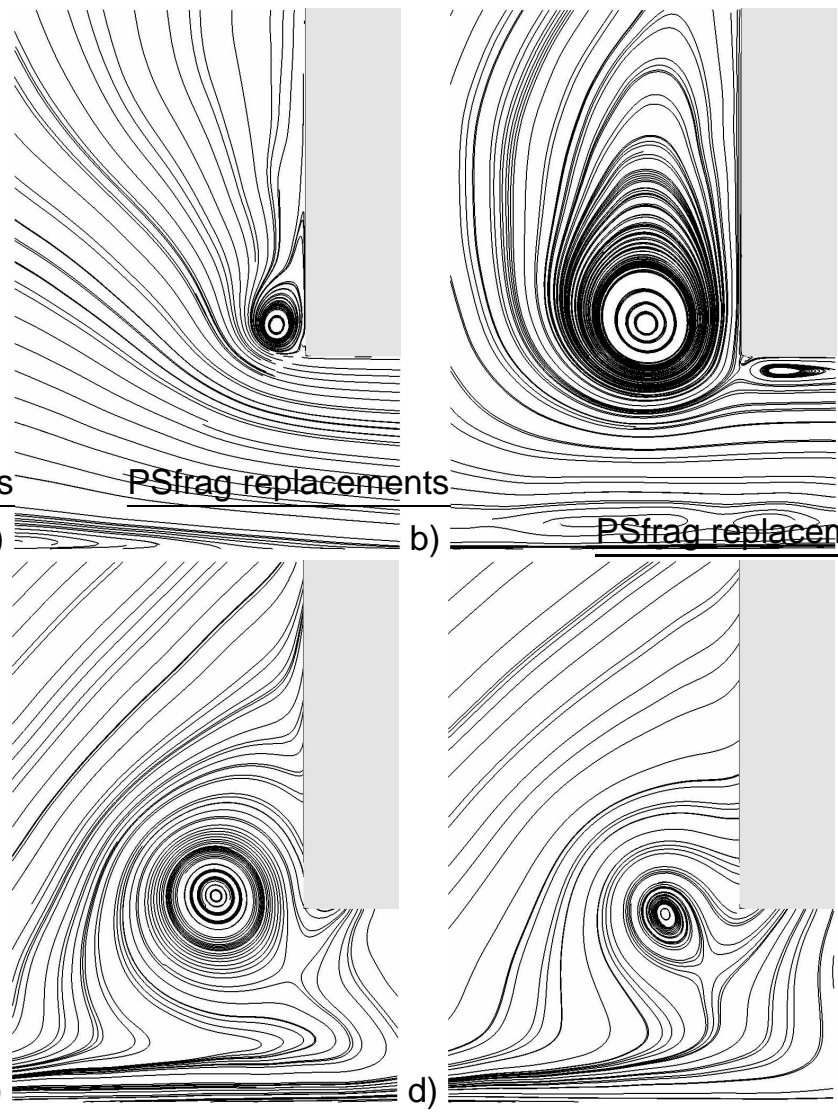

b)

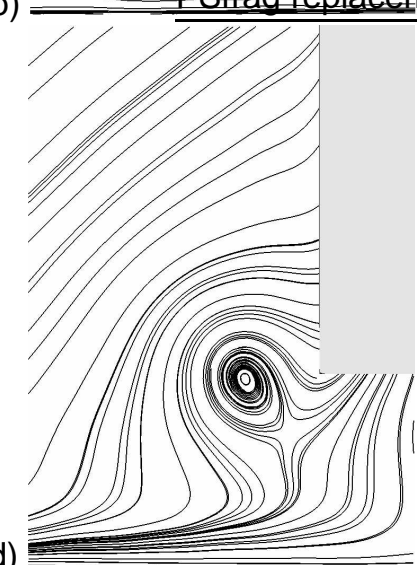

Figure 6: Time-averaged streamlines projected onto planes: (a) $x=-2.72 H$, (b) $x=-0.91 H$, (c) $x=0.17 H$ and (d) $x=0.35 H$. The direction of the rotation of this vortex is clockwise. Front view of the lower-left edge of the body.

shown in Fig. 7. As we see in Fig. 7, the topology of the flow observed in the experiments is accurately predicted in LES. The imprints of the trailing vortices and the spanwise separation region are visible in both the oil-film visualization and the LES. The experimental observation of unstable reattachment line in Fig. 7 a) was confirmed in the LES (Fig. 7 b)).

In both simulations a separation region containing three vortical structures (Figs. 8 b) and c)) in agreement with the experimental observations (Fig. 8 a)) was observed. The same observation is made for the small separation region on the slant surface of the body (Note that this separation region is not shown in Fig. 8 a) but was observed in the experiments by Lienhart and Becker [7].) Surprisingly the under-resolved LES (on the coarse grid) predicted the flow topology that was very close to the one from the medium grid simulation. Although they are similar there are differences in size and shape of the separation regions.
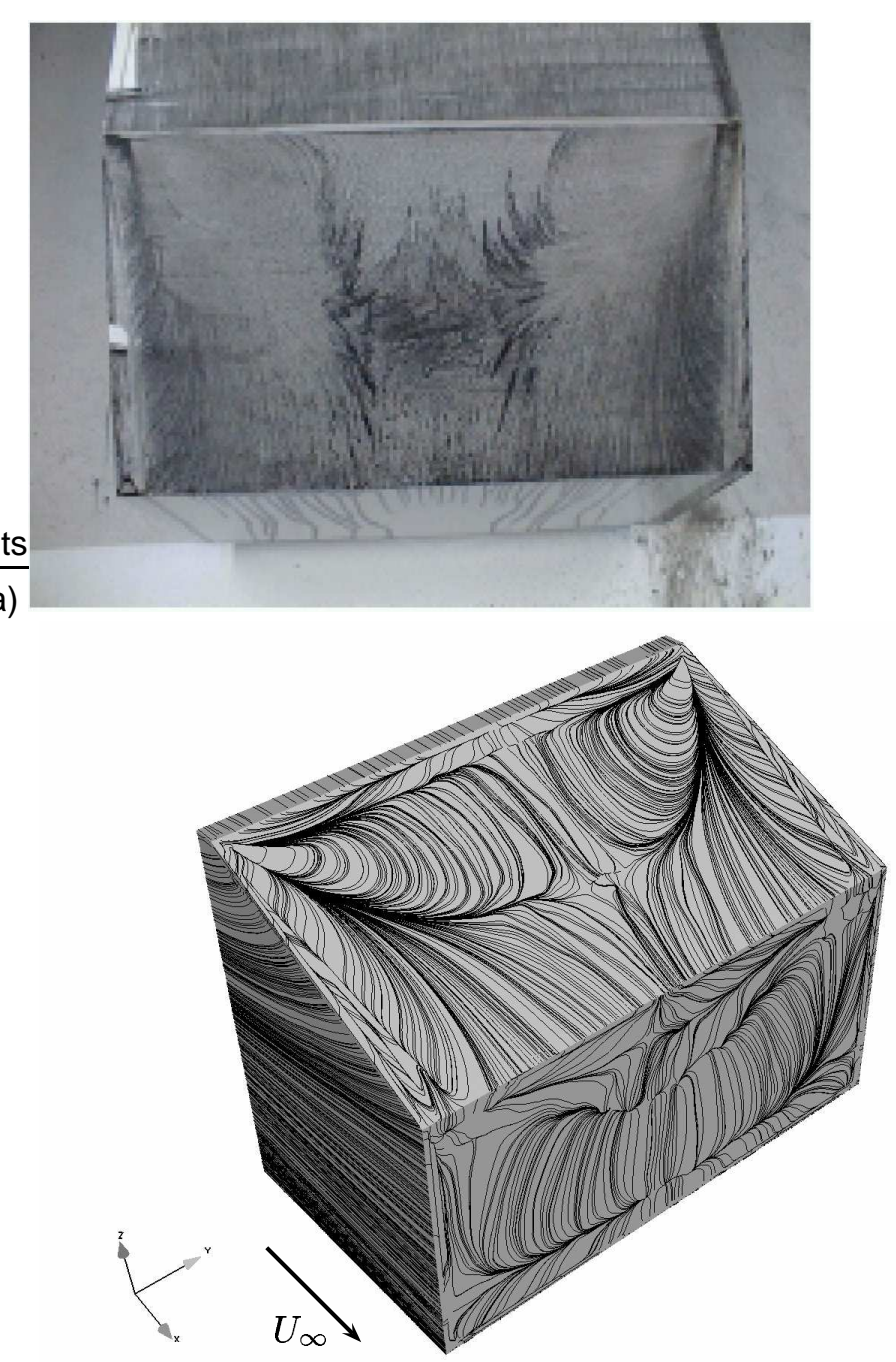

b)

Figure 7: Comparison of a) oil-film visualization by Lienhart and Becker [7] at $R e=7.68 \times 10^{5}$ with b) timeaveraged trace lines on the surface of the body from LES. Only the rear part of the body is shown.View from behind the body.

VELOCITY PROFILES The velocity profiles from LDA (laser doppler anemometry) measurements from [7] are compared with our LES results in Figs. 9 and 10 . The results are presented above the rear slant surface from $x=-0.83 H$ to $x=-0.01 H$ with a distance of $\delta x=0.07 H$ between two profiles. Both simulations give much better agreement with the experimental data than previous RANS and LES simulations from [10]. We find again large similarities in the results for wall-normal velocities $\langle W\rangle_{t}$ between the two simulations in Fig. 9. The differences become more obvious in Fig. 10 where the comparison of the streamwise velocity profiles is presented. Although both simulations do a good job in the separation bubble the coarse grid simulation fails to predict the attached flow downstream and upstream of this region (see Fig. 10). The upstream boundary layer is too thin whereas the downstream attached flow has an 
excess of momentum (see Fig. 10). The medium grid simulation produces a picture of the flow in better agreement with experiments than that with the coarse grid. The upstream boundary layer for the medium-grid simulation is in agreement with the experimental data and the attached flow after the reattachment is accurately predicted. Some differences in the velocity profiles are visible in the region of the separation bubble probably as a result of too coarse a spatial resolution in this re-

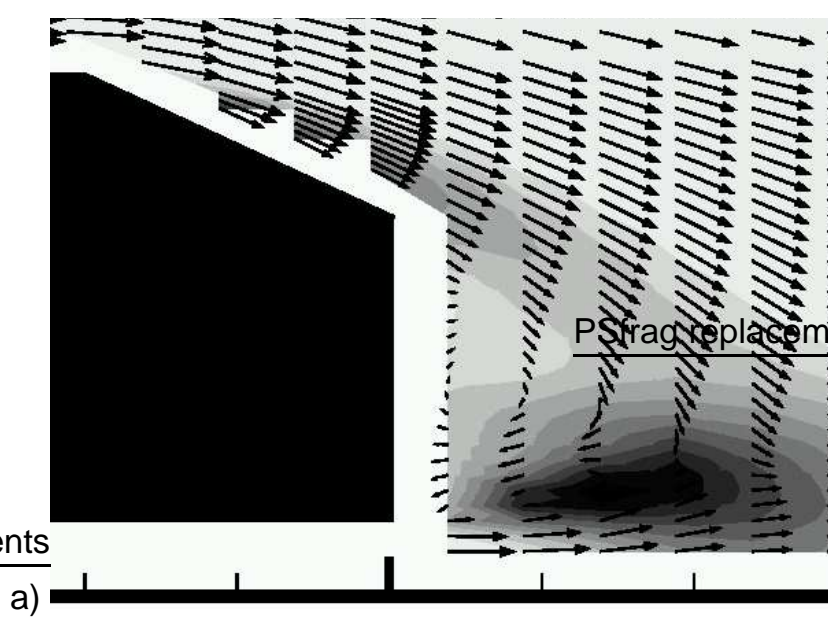

b)
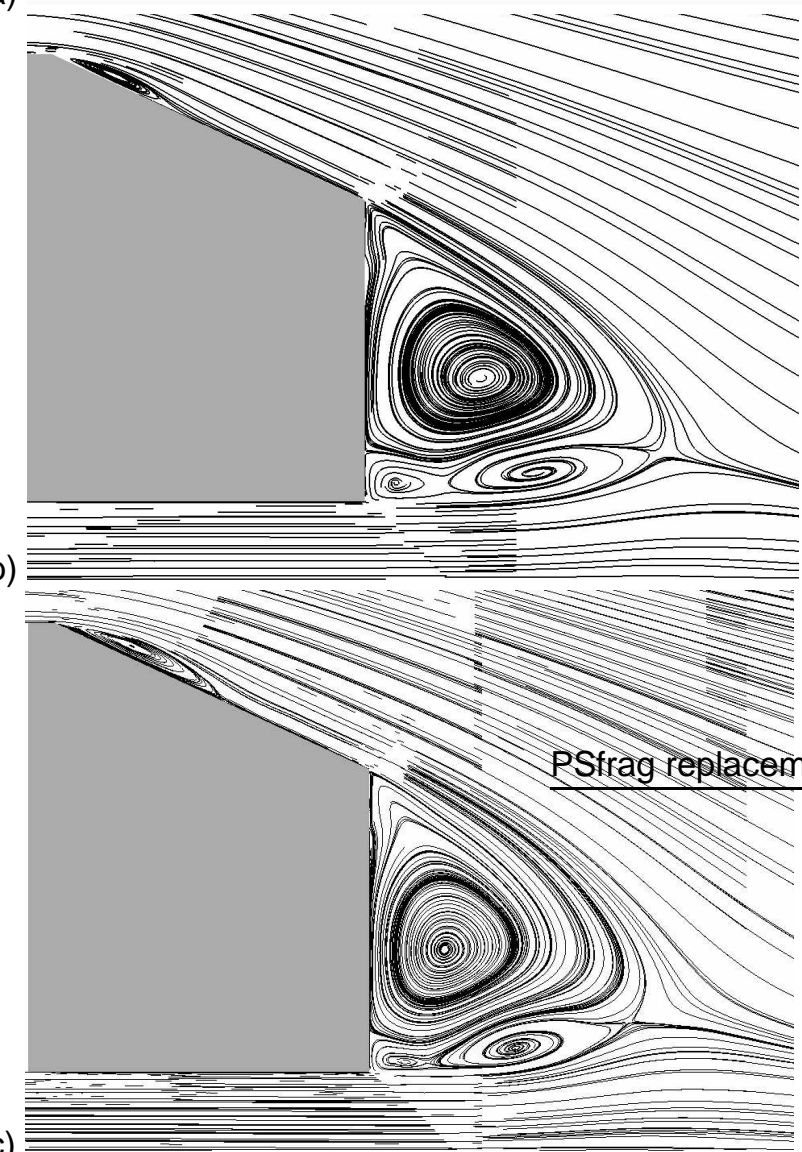

c)

Figure 8: Comparison of (a) the velocity vectors from experiments [7] with the time-averaged streamlines projected onto the symmetry plane $y=0$ of the car body from LES using (b) course grid and (c) medium grid. gion of the flow.

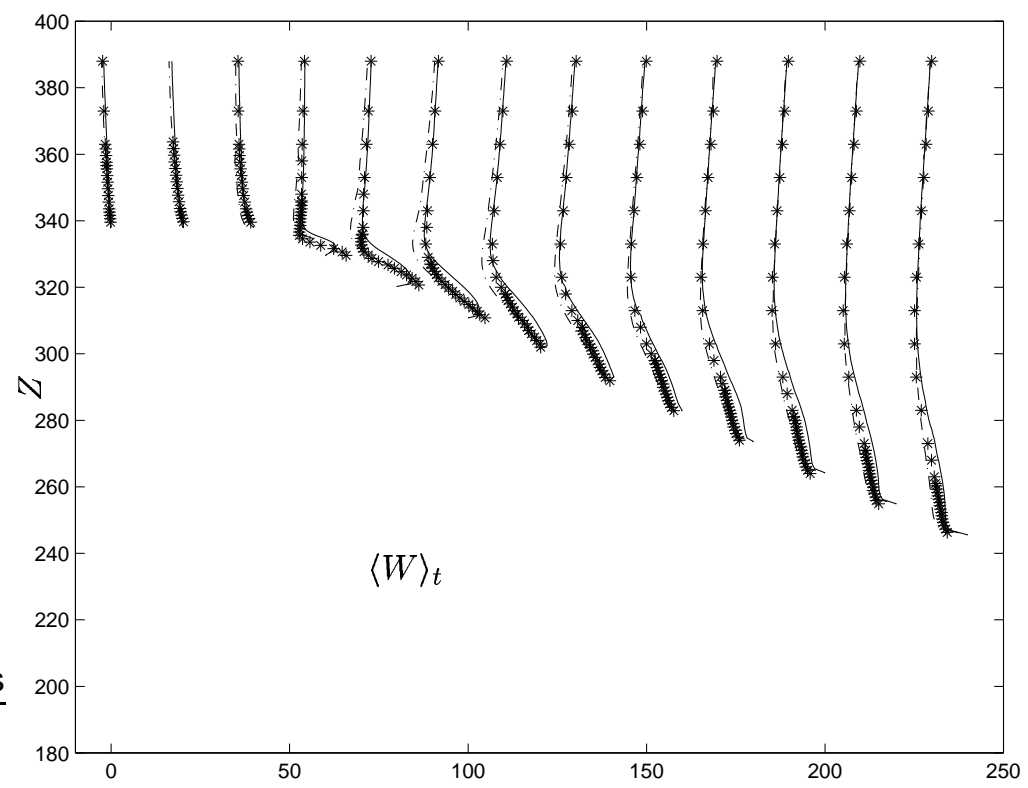

Figure 9: Time-averaged wall-normal velocity profiles in the symmetry plane. Medium grid (solid curve); coarse grid (dashed-dotted curve); experiment (symbols).

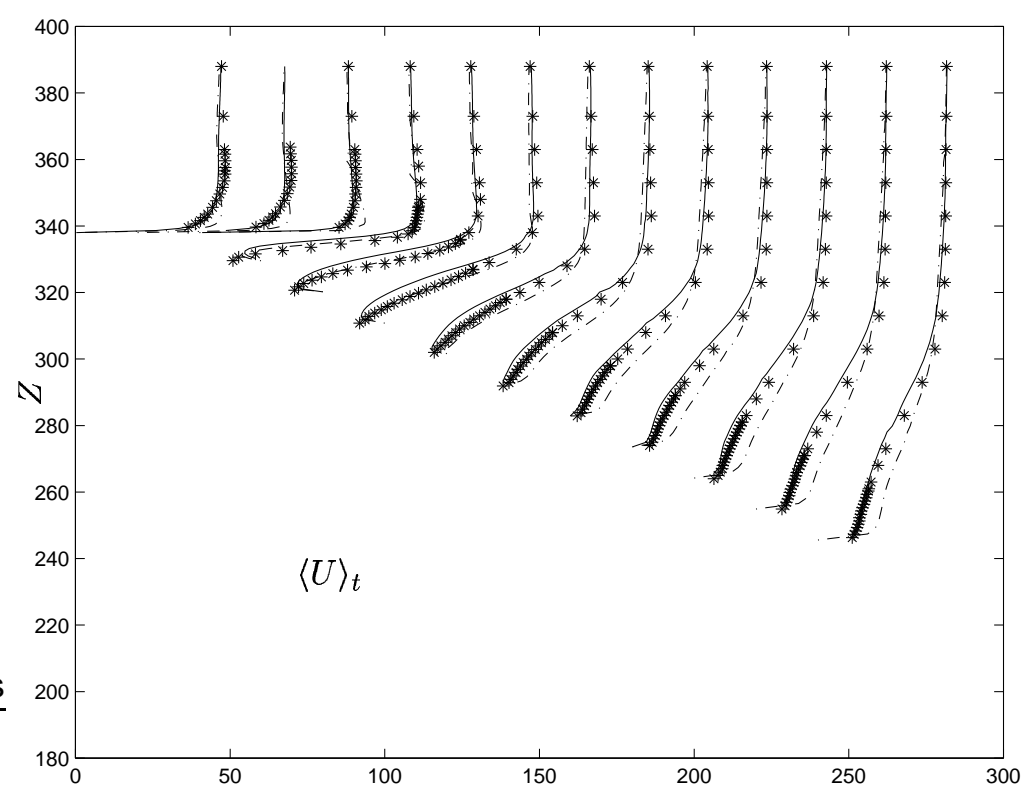

Figure 10: Time-averaged streamwise velocity profiles in the symmetry plane. Medium grid (solid curve); coarse grid (dashed-dotted curve); experiment (symbols).

SURFACE PRESSURE The pressure-coefficient distribution on the rear part of the model from the two LES is presented in Figs. $11 \mathrm{~b}$ ) and c) and compared with the experimental data in Fig. 11 a). The pressure picture on the slant surface is similar in the two simula- 
tions although the medium grid simulation is in slightly better agreement with the experiment. More significant differences are observed on the rear vertical face where the medium grid simulation predicted the imprint of the large upper and small lower separation regions in the wake. The coarse grid simulation fails to predict the pressure drop on the upper part of this surface.

CONCLUSIONS Large eddy simulation using Reynolds number of $R e=2 \times 10^{5}$ resulted in the flow around rear part of the simplified car similar to the one in the experiments at higher Reynolds number $\left(R e=7.68 \times 10^{5}\right)$. These results indicate (at least for the geometry studied in this paper) that the external vehicle flow at high Reynolds number becomes insensitive to the Reynolds number. It seems that the geometry rather than the viscosity dictates the character of the flow (attached or detached) and the position of flow separations. Using lower Reynolds number in our LES we can resolve the near-wall energy-carrying coherent structures and predict the flow accurately. This observation raises hope that flow around real cars can be simulated with LES at reduced Reynolds numbers.

The results of our LES show wide range in size of the turbulent scales around the rear slant surface. This variety in the turbulent length scales in combination with very unsteady reattachment of the separated flow in this region are possible reasons for failure of RANS simulations to predict this flow.

ACKNOWLEDGMENTS This work was supported by the FLOMANIA project. The FLOMANIA (Flow Physics Modelling - An Integrated Approach) is a collaboration between Alenia, AEA, Bombardier, Dassault, EADS-CASA, EADS-Military Aircraft, EDF, NUMECA, DLR, FOI, IMFT, ONERA, Chalmers University, Imperial College, TU Berlin, UMIST and St. Petersburg State University. The project is funded by the European Union and administrated by the CEC, Research Directorate-General, Growth Programme, under Contract No. G4RD-CT2001-00613. Computer time on the Linux cluster, provided by the NSC (National Supercomputer Center in Sweden), is gratefully acknowledged.

\section{REFERENCES}

[1] S. K. Robinson. Coherent motions in the turbulent boundary layer. Ann. Rev. Fluid Mech., 23:601639, 1991.

[2] S. Krajnović. Large Eddy Simulations for Computing the Flow Around Vehicles. PhD thesis, Dept. of Thermo and Fluid Dynamics, Chalmers University of Technology, Gothenburg, 2002.

[3] S. R. Ahmed, G. Ramm, and G. Faltin. Some salient features of the time averaged ground vehicle wake. SAE Paper 840300, 1984.

[4] D. B. Sims-Williams and R. G. Experimental investigation into unsteadiniess and instability in passanger car aerodynamics. SAE Paper 980391, 1998.

[5] I. Bayraktar, D. Landman, and O. Baysal. Experimental and computational investigation of ahmed body for ground vehicle aerodynamics. SAE Paper 2001-01-2742, 2001.

[6] A. Spohn and P. Gillieron. Flow separations generated by a simplified geometry of an automotive vehicle. In IUTAM Symposium: Unsteady Separated Flows, April 8-12, Toulouse, France, 2002.

[7] H. Lienhart and S. Becker. Flow and turbulente structure in the wake of a simplified car model. SAE Paper 2003-01-0656, 2003.

[8] D. B. Sims-Williams and B. D. Duncan. The ahmed model unsteady wake: Experimantal and computational analyses. SAE Paper 2003-01-1315, 2003.

[9] T. Han. Computational analysis of threedimensional turbulent flow around a bluff body in ground proximity. AIAA Journal, 27(9):1213-1219, 1989.

[10] R. Manceau and J.-P. Bonnet. 10th joint ERCOFTAC (SIG-15)/IAHR/QNET-CFD Workshop on Refined Turbulence Modelling. Poitiers, 2000.

[11] E. G. Duell. Experimental investigation of unsteady near wakes of ground vehicle bodies. PhD thesis, Cornell University, 1994.

[12] E. G. Duell and A. R. George. Experimental study of a ground vehicle body unsteady near wake. SAE Paper 1999-01-0812, 1999.

[13] J. B. Barlow, R. Guterres, and R. Ranzenbach. Rectangular bodies with radiused edges in ground effect. AIAA paper 99-3153, 1999.

[14] J. Barlow, R. Guterres, R. Ranzenbach, and J. Williams. Wake structures of rectangular bodies with radiused edges near a plane surface. SAE Paper 1999-01-0648, 1999.

[15] S. Krajnović and L. Davidson. Numerical Study of the Flow Around the Bus-Shaped Body. ASME: Journal of Fluids Engineering, 125:500-509, 2003.

[16] S. Ghosal. Mathematical and physical constraint on large-eddy simulations of turbulence. AIAA Journal, 37(2):425-433, 1999. 
[17] J. Smagorinsky. General circulation experiments with the primitive equations. Monthly Weather Review, 91(3):99-165, 1963.

[18] S. Krajnović and L. Davidson. Large eddy simulation of the flow around a bluff body. AIAA Journal, 40(5):927-936, 2002.

[19] A. Sohankar, L. Davidson, and C. Norberg. Large eddy simulation of flow past a square cylinder: Comparison of different subgrid scale models. ASME: Journal of Fluids Engineering, 122(1):3947, 2000.

[20] A. Sohankar, L. Davidson, and C. Norberg. Erratum. ASME: Journal of Fluids Engineering, 122(3):643, 2000.

[21] L. Davidson and B. Farhanieh. CALC-BFC: A finite-volume code employing collocated variable arrangement and cartesian velocity components for computation of fluid flow and heat transfer in complex three-dimensional geometries. Report 95/11, Dept. of Thermo and Fluid Dynamics, Chalmers University of Technology, Gothenburg, 1995.

[22] H. Nilsson and L. Davidson. CALC-PVM: A parallel SIMPLEC multiblock solver for turbulent flow in complex domains. Internal report 98/12, Department of Thermo and Fluid Dynamics, Chalmers University of Technology, Gothenburg, 1998.

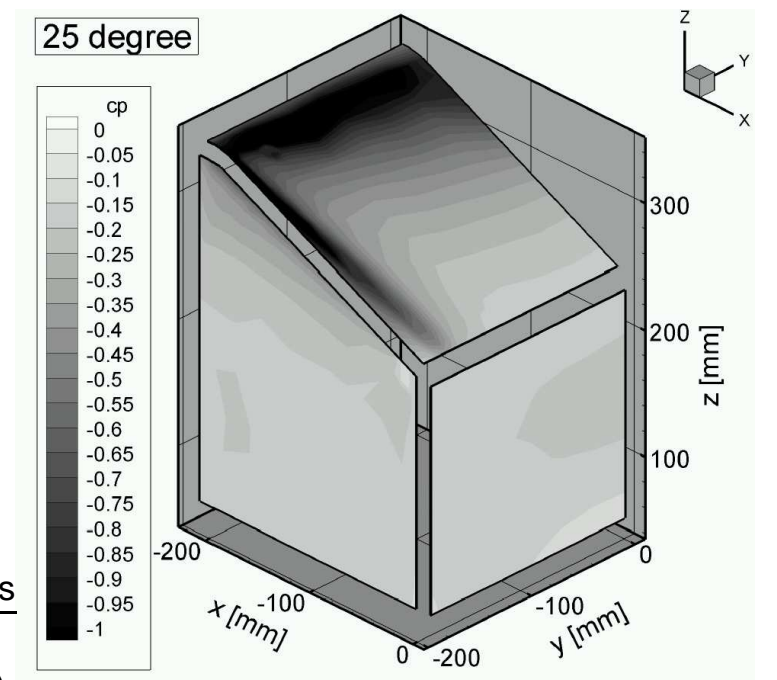

a)

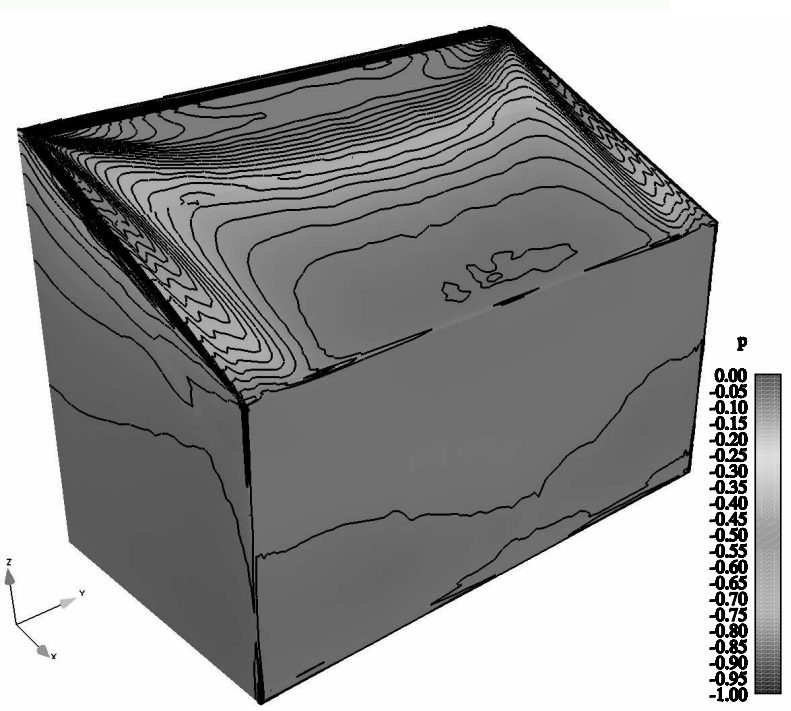

b)

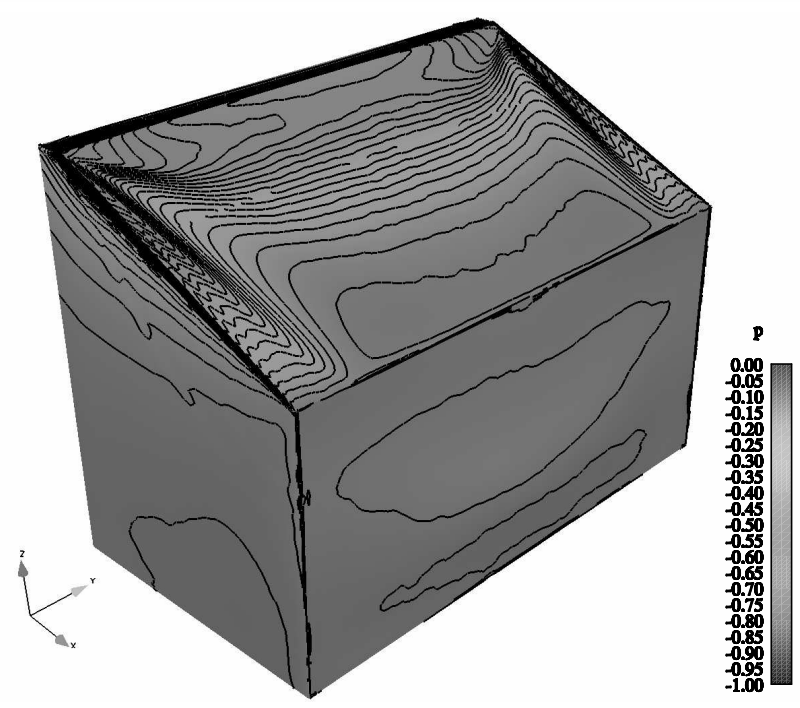

Figure 11: Comparison of the pressure-coefficient distribution on the rear of the model between (a) experiment [7]; (b) LES on coarse grid and (c) LES on medium grid. 